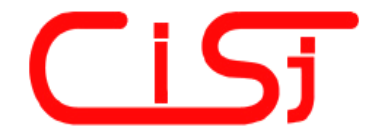

\title{
INFORMATION INTERACTION OF THERMOCHEMICAL POTENTIALS IN LIVING ORGANISMS AND GENERAL THEORY OF RELATIVITY
}

\author{
Volodymyr Tydnjuk ${ }^{1)}$, Volodymyr Kravchenko ${ }^{2)}$, Olena Mul ${ }^{3)}$, Mykola Shut ${ }^{4)}$ \\ 1) International Scientific and Educational Center of Information Technologies and Systems, \\ 40 Academician Glushkov avenue, $03680 \mathrm{Kiev}$, Ukraine \\ ${ }^{2)}$ Institute of Cybernetics named by V. Glushkov, 40 Academician Glushkov avenue, 03680 Kiev, Ukraine \\ ${ }^{3)}$ Institute of Computer Information Technologies, Ternopil Academy of National Economy, \\ 3 Peremoga Square, 46004 Ternopil, Ukraine, omu@tanet.edu.te.ua \\ 4) National Pedagogical University named by M. Dragomanov, 9 Pirogov str., 01601 Kiev, Ukraine
}

\begin{abstract}
The application of general theory of relativity for building of model of living organisms field is considered. The information field, which connects living organism with environment, forms a material object of such field. A nonelectromagnetic nature of such material object is substantiated. The phenomenon of interaction of thermochemical potentials at distance is investigated.
\end{abstract}

Keywords: - general relativity theory, living organisms information fields, thermochemical potentials interaction

\section{INTRODUCTION}

Nowadays the fact of existence of fields with different physical nature around bio-objects is undoubted. But the deep research of such fields during last decades generated much more questions and problems than it were initially. First of all, the problem is how to differentiate passive fields and fields with active information loadings during activity of ferment and physiological systems of organism. Further if the existence of some "purely information" field is accepted then the material object of such a field, and channels of action of slight information signal onto physicochemical processes in organism etc. should exist.

Recently a lot of different information technologies have been developed, which use complex technical devices with corresponding computer hardware and software. Such devices first affect a bio-field of organism and then physiological systems of organism as well. For that both irradiation by electromagnetic fields of different spectral regions and irradiation by other specific coercions are applied. Such influences take place on microorganisms inside tissues and organs. Also the influences take place on aqua or other neutral supporters, whereupon such supporters have a possibility of the information influence on organism.
But all these technologies have either completely empirical character or absolutely non-adequate theoretical substantiation. Moreover, the most of such information technologies are in use but they are not described in a scientific literature and have some know-how's because of commercial reasons. A lack of the knowledge about material object of information signal and characteristics of its influence on physical-chemical processes in organism may bring to the fact that application of such technologies may have some negative consequences. Because of a weak information effect, such consequences may appear not at once after the influence but much later.

Systematization of the known empirical facts and an attempt to find their logical relation with present physical theories indicate that deep reconstruction of space-time properties, which may be described with a help of general theory of relativity, corresponds the most adequately to the role of the material object of bio-objects information field $[1,2]$. The equations of this theory describe actually not only gravitation generated by mass density distribution but as well any other field, for which any form of energy may be a source including chemical reactions energy.

In 2005 it will be 100 years after creation of special theory of relativity, and in 2016 it will be 100 years after construction of mathematical model 
of general theory of relativity by Einstein. Fundamentality of ideas of these theories allows drawing several conclusions of methodological character at least.

Firstly, all the fields of applications of relativity theories are not investigated so far. Secondly, some specific fallacies exist concerning interpretation, and universality, or, on the contrary, absolute and unfounded denial of these theories. Thirdly, after 100 years of progress of theoretical physics, and mathematics with its applications in topology, general theory of communication, and systems analysis, it is obvious, that both relativity theories are approximate the same way as all theories, and they need a further development.

Besides, the formulas of special theory of relativity are applied everywhere, for example, in atomic physics, or in elementary-particle physics, but none technical device has not been created on the base of general theory of relativity. However, this theory has a theoretical application, such as models universe of Freedman, or computation of Mercury orbit characteristics, and others.

\section{SOME FREQUENT INACCURACIES OF GENERAL RELATIVITY THEORY INTERPRETATION}

The basic idea of general theory of relativity is that density of any energy form changes space curvature and metric, i.e. space structure. This idea is fixed in general form of basic equation of general relativity theory [3]:

$$
\alpha \mathrm{T}_{\mathrm{ij}}=\mathrm{S}_{\mathrm{ij}} \text {, }
$$

where $T_{i j}$ is energy-momentum tensor, $S_{i j}$ is construction from curvature tensors and metric tensor, $\alpha$ is numerical coefficient.

Solving of such equations in pseudo-Euclidean space of Riemannian geometry implies some difficulties and complexity of interpretation, therefore certain fallacies and discrepancies still occur in this regard in applications of ideas of general relativity theory.

First of all, it follows from equations (1) that all energy forms, changing space structure, generate some unified field against vacuum. But it does not follow from this the possibility of creation of unified field theory. So, if two forms of independent energies of physical interaction exist, then insertion of densities of these energies into equations (1) implies that these forms will interact for the present problem with possible transformation of energy forms from each other.
At the same time, in most cases independent energy forms do not interact, therefore it is necessary to write a separate structure of equations system (1) for each of them. Thus, it is possible to describe, for example, gravitational interaction with a help of equations like (1). However, the EinsteinMaxwell equations [4] describe quite different range of problems regarding interaction of electromagnetic radiation with gravitation and space.

Therefore, in terms of general theory of relativity one may consider some "unified field" but it is a significant fallacy to try to build "unified field theory" practically, when all forms of interactions are represented with a help of equations set (1) and they become dependent in fact. In essence, the concrete formulation depends only on the range of problems, which are solved with a help of the equations of general relativity theory. Furthermore, Einstein brought up a question about "unified field theory" in a different sense. He was interested in a possibility of representation of any fields, including strong interaction, "in a geometrical way", as variations of a space structure, which are described by means of Riemannian geometry.

\section{ARGUMENTATION OF NON- ELECTROMAGNETIC NATURE OF FIELD, CONNECTING LIVING ORGANISM WITH ENVIRONMENT}

The hypotheses of electromagnetic origin of organism information field are criticized in the paper [2]. In fact such a field exists in different spectrum regions but nevertheless it carries passive load only and can not be a signal transmitter thanks to which any action onto biologically active points (to be named BAP) results in changes of functioning mode of corresponding organs and physiological systems.

A number of other theories exist also but their adequacy will not be discussed in this paper.

Here let us consider some features of nonelectromagnetic hypothesis about physical essence of field, which connects internal organs and systems, BAP and environment. Let us give the equation of Einstein general relativity theory in the full form [3]:

$$
\alpha \mathrm{T}_{\mathrm{ij}}=\mathrm{R}_{\mathrm{ij}}-\frac{1}{2} \mathrm{Rg}_{\mathrm{ij}}
$$

where $T_{i j}$ is energy-momentum tensor, $R_{i j}$ is Richy tensor (convolution, trace of curvature tensor), $\mathrm{R}$ is scalar curvature in pseudo-riemannian space, $g_{i j}$ is metric tensor, $\alpha$ is numerical coefficient.

The energy of chemical reactions is the leading form of energy in living organisms. In this case we 
can consider that Gibbs energy or some similar thermochemical potential generates space curvature and change of its metrics. From a phenomenological analysis of the equation (1) it is obviously that two spaced chemical reactions should affect on each other by means of change of curvature and vacuum metrics.

Gibbs energy is connected with so called electrolytic potential:

$$
-\Delta \mathrm{G}=\mathrm{nFE},
$$

where $\mathrm{n}$ is a number of equivalents of participating in the reaction substances, $F$ is Faraday constant, $\mathrm{nF}$ is equal to quantity of passed electricity, $\mathrm{E}$ is electromotive force.

In fact, the changes of Gibbs energy bring to the changes of such environment electroconductive characteristics as ions quantity and electric field strength, especially in electrolytes, even if the components of direct galvanic transformation into electric energy are absent.

In electrolytes ions quantity increases at external increasing of electric field strength, therefore the change of electric conductivity is here a driving member at changes of Gibbs potential. Thus, a presence of curvature-metrics field explains a change of electroconductive characteristics in a point, which is distant from a zone of chemical reaction. As a matter of fact, exactly this effect is used in electro-acupuncture practice.

Situated on skin, BAP changes its electric conductivity depending on intensity of chemical reactions in heart, liver, or other organs. On the other hand, changing Gibbs energy in situated on skin BAP in such way as acupuncture, pressure and thermal effects, electro-acupuncture, laser therapy, etc., we can influence on intensity of chemical reactions in internal organs. The changes of electric conductivity are observed not only in isolated points but also in some areas, so called Zaharjin-Ged zones.

In paper [2] the estimation of constant of thermochemical potentials interaction is given on the base of equations of general relativity theory:

$$
\mathrm{k}_{\mathrm{t}} \approx 10^{5} \mathrm{k}_{\mathrm{g}}
$$

where $k_{t}$ is constant of thermochemical potentials interaction, $\mathrm{k}_{\mathrm{g}}$ is constant of gravitational interaction.

In SI system constant of gravitational interaction is $\mathrm{k}_{\mathrm{g}} \approx 6.67 \cdot 10^{-11}$. Thus, if gravitational interaction is about $10^{-11}$ of energy-radiator density, then thermochemical interaction may be $10^{-6}$ of radiating process power. Such not large power confirms an information character of vacuum metric field, which is generated by Gibbs energy. But equations of general relativity theory do not give a direct answer on the next question: how does metric information effect change the parameters of thermochemical process?

Here we deal not even with a specific character of interaction between fields of different types and living organism, but also with an existence of some general phenomenon of information interaction at physical interactions of different types. In order that ultra-small signal changed a state of material system step-wise, this signal should "know" "controlling chains", and points of system instability, etc. The most realistic approach is search of such mechanism in deep time properties that allows in some way to connect past, present and future events, which probably do not interact at present but which will have (or had) some causal-investigatory relationships.

From this point of view, Einstein modeling of time properties in both theories of relativity with a help of the fourth space coordinate, even an imaginary one, is approximate and rather inexact. Time is most probably a factor, which in the small destroys and changes space structure, connectedness, and other topological characteristics.

The approach developed in quantum mechanics seems to be more accurate. Here non-normalized probability $\mathrm{p}$ of every physical event with microobject is connected with so-called wave function:

$$
\begin{gathered}
\psi=\varphi_{1}(\mathrm{x}, \mathrm{y}, \mathrm{z}, \mathrm{t})+\mathrm{i} \varphi_{2}(\mathrm{x}, \mathrm{y}, \mathrm{z}, \mathrm{t}) \\
\mathrm{p}=\psi \psi^{*}
\end{gathered}
$$

Such an approach is interpreted a priori only, as a convenient mathematical construction, from which correct equations of motion follow. But here the next question is not considered: why is it necessary to use complex, but not real function of space coordinates and time for calculation of events probabilities? Actually, such model may be interpreted as an existence of virtual or real parallel space and fine spaces interaction, which causes a phenomenon of information effect for remote along time axis events.

Thereupon, let us consider the theory of Polish physicist T. Kalutsa. From extended later versions of his theory it follows that it is possible to describe not only gravitation but also absolutely all physical interactions against vacuum, both electromagnetic weak ones, and strong ones, with a help of general relativity theory as phenomenon of change of structure, curvature, metrics, torsion, and other 
characteristics of space. But for that it is necessary to apply general relativity theory not to the threedimensional space with time as the fourth coordinate but to the ten-dimensional space with time as the eleventh coordinate.

Kalutsa himself started from consideration of five-dimensional space and used it for "geometrical $"$ description of electromagnetic forces. Naturally, "unnecessary " space dimensions were the subject for criticism in Kalutsa theory as in fact we observe three-dimensional universe. The first serious attempt to cope with this difficulty was made by Swedish theorist O. Klein.

In his opinion, introduced by Kalutsa spare space dimensions really exist. We do not sense them just because universe in this direction has a microscopically small radius, i.e. it is a tiny closed circle with a radius of the order of $10^{-33} \mathrm{~cm}$. Klein gave a water-pipe as an example: it seems to be a one-dimensional line from a distance, but closely we can see two more its dimensions.

But he did not write anything about possibility of existence of complementary dimensions not in the physical world but in parallel spaces, and about their virtual, i.e. for a very short time, interaction. Apropos, complementary unknown for us forces of interaction should exist in multidimensional spaces, and this was not accounted in the attempts to build "specific physics" of multidimensional space independently on collisions around general relativity theory [5].

But prior introduction of complex wave function for description of events possibilities in quantum mechanics allows returning to the idea of qualitatively other physical world, in which at least virtually multidimensional parallel spaces are possible. Complex wave function may be interpreted as "four-dimensional junction" of such spaces as simultaneous interaction of two three-dimensional cuts takes place in $(\mathrm{x}, \mathrm{t})$ point. Such cuts are correspondingly described with a help of real and imaginary parts of wave function, and interaction of these cuts results in change of possibilities of physical events.

Thus, time phenomenon should be considered more thoroughly, and modeling of time characteristics by complementary space-similar coordinate is a very rough approximation. Introduction of multidimensional parallel spaces and their virtual contact is also only mathematical abstraction without pretention about physical nature hypotheses. But such interpretation seems to be more effective in comparison with a classical statement when wave function of elementary particle or atom is described in multidimensional Hilbert space whereas in fact the real space is threedimensional.

From this point of view wave function of probability may also be considered as a suitable one for description of macro-events. Let us consider a particle on plane. At some moment the point receives an impulse with probability of deviation along polar angle $\alpha$, which has a normal distribution law around mean $\alpha=a$, i.e. the real part of wave function $\psi$ is the next:

$$
\varphi_{1}=\frac{1}{\sigma \sqrt{2 \pi}} \mathrm{e}^{-\frac{(\alpha-\mathrm{a})^{2}}{2 \sigma^{2}}}
$$

Let us assume that at some point of plane there is the second particle and the impact is possible in motion. In this case the imaginary part of wave function should contain the probability of this event, i.e. impact occurrence. Thus, accounting the imaginary part, the most acceptable form for wave function will be:

$$
\psi=\frac{1}{\sigma \sqrt{2 \pi}} \mathrm{e}^{-\frac{(\alpha-\mathrm{a})^{2}}{2 \sigma^{2}}}+\mathrm{i} \frac{1}{\sigma \sqrt{2 \pi}} \mathrm{e}^{-\frac{(\alpha-\mathrm{b})^{2}}{2 \sigma^{2}}}
$$

where $b$ is mean of impulse direction after impact in the case of impact, $\sigma$ is variance.

Then distribution density of direction of particle impulse motion is the next:

$$
\begin{aligned}
\mathrm{p}=\psi \psi^{*}= & \frac{1}{\sigma \sqrt{\pi}}\left[\frac{1}{\frac{\sigma}{\sqrt{2}} \sqrt{2 \pi}} \mathrm{e}^{-\frac{(\alpha-a)^{2}}{2\left(\frac{\sigma}{2}\right)^{2}}}+\right. \\
+ & \left.\frac{1}{\frac{\sigma}{\sqrt{2}} \sqrt{2 \pi}} \mathrm{e}^{-\frac{(\alpha-b)^{2}}{2\left(\frac{\sigma}{2}\right)^{2}}}\right]
\end{aligned}
$$

Probability density $\mathrm{p}$ in expression (8) has a local maximum at points $\alpha=a$ and $\alpha=b$, and it is a double-humped distribution of stochastic variable.

In case of absence of the second particle, the imaginary part of wave function may be considered as equal to unit, or as uniformly distributed on the interval $(0 ; 2 \pi)$, that will model friction presence.

The most of the macro-events are deterministic, and it seems that application of probabilistic values of parameters must not affect such events. But variations of probabilities of values may have an influence on the behavior of the higher derivatives 
of the process function. So only derivatives of the first and second order are deterministic within definite limits and exactly these derivatives are present in the known macroscopic equations of motion. Thus, a probabilistic variation of the higher derivatives leads gradually to the variation of the whole function of the process, which was considered as a deterministic one. Actually, the influence on the higher derivatives of the function, which describes some parameters of a physical or chemical process, is informative, i.e. it is small in comparison with process energy but controlling regarding its basic parameters.

Integrating phenomenon of distant influence of thermochemical potentials and phenomenon of their information effect along time axis, which are above investigated theoretically, we may expect the next:

(i) thermochemical reaction, for example reaction of diffusion of solution coloration, should mainly have a normal distribution regarding its parameters;

(ii) if during reaction we put the second reservoir nearby with one more thermochemical reaction inside, or in some other way change natural Gibbs potential of solution at the distant moment from reaction start, then the second hump may appear in parameters distribution, i.e. two points of local maximum in distribution of probability density may take place.

The fact of existence of double-humped distributions was found by S. Shnol' during experiments on both animate and inanimate substrates [6], [7]. It was found that rate of chemical reactions, sedimentation of solution, fungus growth depend definitely on place and time and are not explained by some fluctuations. In fact, some waves of indeterminate origin were found, which had an influence on physicochemical processes both in animate and in inanimate substrates.

The experiments with famous actinomycetes were completely unique. They were carried out aboard ships, airplanes, satellites, and even space complex "Sojuz-Apollon". Rate of fungus increase was dependent on geographical coordinates, angular velocity relative to the Earth and oscillations of its magnetic field.

Thus, for example, Shnol' investigated classical reaction of hydrolysis of energy-containing ATP under the influence of muscular ferment. There was a dispersion of the results, and deviations divided into two groups with a large break between them. Their form at a graph was not a rough bell surface simply, but it was a double-humped one, and that contradicted the classics.

At that time explanation of theoretical essence of such experiments had a single meaning: it was influence of solar activity and geomagnetic field of the Earth. However such correlation should be proved also by experience, but equipment for measurement of little changes of geomagnetic field parameters exists only at present. Moreover, the most of changes of geomagnetic field has large periods, but many of the experiments in Shnol' series are short-term.

So Shnol' experiments may be just indirect proof of above-stated theoretical constructions. But even if it fails to find direct experimental proof that will mean only the next: equations of relativity theory represent not all of the physical factors, they are approximate and need modifications.

We may expect that wave function for macroevents of the type (7) should satisfy the analog of Shroudinger equations [8]:

$$
\begin{aligned}
& -\frac{\mathrm{h}^{*}}{\mathrm{i}} \frac{\partial \psi}{\partial \mathrm{t}}=-\frac{\mathrm{h}^{*}}{2 \mathrm{~m}_{0}} \nabla^{2} \psi+\mathrm{V} \psi \\
& \frac{\mathrm{h}^{*}}{\mathrm{i}} \frac{\partial \psi^{*}}{\partial \mathrm{t}}=-\frac{\mathrm{h}^{*}}{2 \mathrm{~m}_{0}} \nabla^{2} \psi^{*}+\mathrm{V} \psi^{*},
\end{aligned}
$$

where $\mathrm{m}_{0}$ is generalized mass of system or particle, $\mathrm{V}$ is its generalized potential energy, $\nabla^{2}$ is Laplacian.

Equations (9) give the possibility to trace the development of wave function with time. But in the case of macro-events it's not possible to interpret constant $h^{*}$ as Planck's constant. First of all it is necessary to determine it experimentally, for example, under the condition of confirmation of experiments, which are analogous to experiments of Shnol'. It is also necessary to expect that this constant is different for interactions of different types, for example, for mechanical one, or for interaction of thermochemical potentials.

Thus, any physical interaction may be accompanied by a weak information interaction along time axis. The last interaction is realized in the form of macroscopic wave function of probability, which is similar to the wave of de Broil for microparticles.

\section{SOME CHARACTERISTICS OF THERMOCHEMICAL POTENTIALS SPACE INTERACTION}

Let us write equation (2) for Gibbs potential in spatially similar coordinates $(\mathrm{i}, \mathrm{j}=1,2,3)$ at some biologically active point $\mathrm{M}$, which is distant from influencing on it zones of chemical reactions in corresponding to this BAP organs and tissues:

$$
\alpha \mathrm{G}_{\mathrm{ij}}=\mathrm{R}_{\mathrm{ij}}-\frac{1}{2} \mathrm{Rg}_{\mathrm{ij}} .
$$


Let us differentiate (10) on time. It is assumed that structure of tensor $G_{i j}$, which corresponds to Gibbs potential, is similar to structure of tensor for mass flow [3]. Then its spatially similar components should be proportional to products of projections of thermochemical potential change rates. These rates may be considered as quasi-constant in the case of absence at point $\mathrm{M}$ of its substantial biochemical activity, i.e. let us accept $\frac{\partial \mathrm{G}_{\mathrm{ij}}}{\partial \mathrm{t}}=0$. Here we take into account also that space influence of distant zones of biochemical reactions is insignificant in comparison with Gibbs potential directly at point M. Practically we restrict ourselves just by analysis of quasi-constant component under interaction of thermochemical potentials. In this way we have:

$$
\frac{1}{2} \mathrm{R} \frac{\partial \mathrm{g}_{\mathrm{ij}}}{\partial \mathrm{t}}=\frac{\partial \mathrm{R}_{\mathrm{ij}}}{\partial \mathrm{t}} .
$$

Metric tensor $\mathrm{g}_{\mathrm{ij}}$, as is well known, is a scalar product of basis vectors of frame for curvilinear system of coordinates corresponding to point $\mathrm{M}$. As equation (10) is written in components of Gibbs potential, changes of metric tensor components with time mean that both Gibbs potential and forces of intermolecular and intramolecular interaction will change in a corresponding way. The last follows from the fact that changes of lengths in local coordinate system as well as appearance of corresponding accelerations are actually equivalent to appearance of complementary forces, which just bring to changes of interaction forces between molecules and their parts. The appearance of such forces is caused by influence on point $\mathrm{M}$ of zones of chemical reactions, which are distant from this point. But change of interaction forces results in the fact that rate of molecules dissociation into ions, ions number, and electric conductivity at point $\mathrm{M}$ should change correspondingly.

Let us consider now a physical interpretation of change of Richy tensor $\mathrm{R}_{\mathrm{ij}}$ with time. It is a convolution of curvature tensor. But geometrical sense of curvature tensor is that its components are proportional to angles of deflection from eigenstate for vector, which traced in a parallel way around closed infinitesimal path [3]. Curvature tensor in the case of Gibbs potential is nonzero at least because rotation part is present in thermal motion of molecules. But it is necessary to expect also other, "more macroscopic" rotation components in thermochemical potential inside of zone of chemical reaction, which influences point $M$ spatially. Such components may appear because zone of chemical reaction fills much more place in comparison with a size of one molecule and often such effect as spatial coherence takes place.

Thus, changes of curvature tensor, and Richy tensor correspondingly, at point $\mathrm{M}$ result both in changes of rotation part of thermochemical potential, and in changes of other fields, which have a nonzero rotor, in particular, of magnetic field. Rotation part of thermal motion corresponds to radiation in millimetric electromagnetic wave band. But usual chaotic thermal motion gives radiation in infra-red band.

Therefore changes of metric tensor cause corresponding fluctuations of electric conductivity and thermal radiation in infra-red band at point $\mathrm{M}$. But changes of curvature tensor result in corresponding fluctuations of magnetic field and radiant intensity in millimetric band (extremely-high frequencies, EHF-radiation). Equation (11) predicts practically a total linear correlation of electric conductivity, magnetic field, radiation in infra-red and millimetric bands at BAP.

The authors of this paper verified correlation of fluctuations of magnetic field and electric conductivity experimentally using Superconducting Quantum Interferometrical Data Unit (SQUID). At that both signal from device, measuring electric conductivity at BAP, and signal from SQUID, directed to the same BAP, were simultaneously received through several channels by corresponding programs at computer for processing.

Software of used for experiments unit SQUID was developed on the base of application-specific program package of the firm "SQUID", Germany. This software allows: making records with corresponding amplitude values, which are simultaneously received through several channels, in a specialized database; noise filtering; spectrum analysis on linear and logarithmic scale; computation of correlations between signals etc.

But now let us consider the same situation under hypothesis of absence of thermochemical potentials interaction. In this case magnetic field and its fluctuations will be induced only by rotational currents in the region of point $M$, which are tangential relative to plane of magnetic field sensor [9]. Electro-neutral flow, for example, the flow of blood or lymph, does not cause magnetic field.

The causes of currents appearance in the region of point $\mathrm{M}$ are the next: cardiosignal; potentials of brain; potentials of stressed, i.e. working muscles; transmembrane currents and nerve impulses in the region of point $\mathrm{M}$. Generally, any current dipole in the region of point $\mathrm{M}$ will cause weaker "volume" currents on any plane around it. 
Cardiosignal is the most powerful among abovementioned causes. But first of all it causes electric field, which is distributed over the whole organism. But electric conductivity differs in different regions and in different directions, besides it changes with time. Therefore electric field is realized as a rule by several or one current dipole. Direction of such dipole does not always coincide with direction of electric vector of heart, as volume character of electric conductivity in the region of point $M$ is quite different from the character in the region of heart. Taking into account the composition with other current dipoles, for which the causes of appearance were mentioned above, it is possible to expect a random signal. Besides mostly we can expect their mutual cancellation that means actual absence of own magnetic field at point $M$, if this point is not close to the regions of heart, brain, stressed muscle, or some other source of not-random currents.

This, in fact, explains that it is necessary for cardiosignal detection to measure not electric conductivity but voltage, moreover differentiated from two points signal is used.

But even if tangential currents, which create magnetic field, are present, it is very complicated to represent their linear correlation with change of conductivity in radial direction in the presence of many independent electric field sources. Thus, it is more likely to think that interaction of thermochemical potentials at a distance is a basic cause of appearance of quasi-constant slowly changing component of magnetogram for some zone of skin surface, especially if this zone is far from heart, brain, or stressed skeleton muscle. As far as correlation of electric conductivity and magnetic field at BAP was verified both theoretically on the basis of equations (11) analysis, and experimentally, BAP magnetogram may be used also as informative signal together with others on purpose to determine states of physiological systems and organs.

The slowly changing component was found earlier in magnetogram but its behavior is not much studied. Moreover an opinion exists that just working skeleton muscle brings to an appearance of this component though it was observed even after muscles work [9]. But quasi-constant component is present in any zone and is correlated with electric conductivity independently of voltage presence in neighboring skeleton muscles.

\section{CONCLUSION}

Thus, actually two independent hypotheses were investigated above such as hypothesis about space interaction of thermochemical potentials and hypothesis about their information interaction along time axis, which may bring to changes of probabilities for some parameters of chemical reactions. It is obvious that a maximum interaction of thermochemical potentials at a small interaction constant occurs when both types of thermochemical potentials interactions have resonance. Such event is the most probable for signals, which are cyclically repeating, and for quasi-constant signals.

\section{REFERENCES}

[1] V. Tydnjuk. F. Gorin. V. Kravchenko. B. Shevchuk. N. Shut. A. Kozakul. On Possibility of Living Organisms Field Modeling with the Help of General Theory of Relativity. Scientific Proceedings of National Pedagogical University named by M. Dragomanov, Physico-Mathematical Science Series. Kiev, 2002, pp. 105-112.

[2] V. Tydnjuk. F. Gorin. V. Kravchenko. B. Beregovskij. B. Shevchuk. On Problem of Information Model in Theory of Living Organisms Fields. Problems of Control and Informatics (2) (2003) pp. 145-152.

[3] P. Rashevskij. Riemannian Geometry and Tensor Analysis. Nauka, Moscow, 1967. p. 664.

[4] N. Mitskevich. Physical Fields in General Theory of Relativity. Nauka, Moscow, 1969. p. 323.

[5] G. Gorelik. Why Is Space Three-Dimensional? Nauka, Moscow, 1982. p. 166.

[6] M. Hromchenko. Space Rhythm of Life. ZnanieSila, Znanie, Moscow, 1986, pp. 27-30.

[7] Biological Timer. Edited by S. Shnol'. Nauka, Moscow, 1964. p. 318.

[8] A. Sokolov. I. Ternov. V. Gukovskij. Quantum Mechanics. Nauka, Moscow, 1979. p. 528.

[9] V. Vvedenskij. V. Ogogin. Ultrasensitive Magnetometry and Bio-Magnetism. Nauka, Moscow, 1986. p. 199.

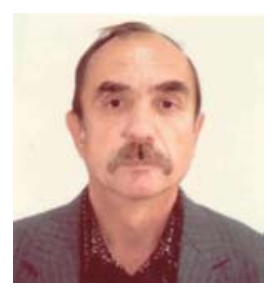

Tydnjuk Volodymyr Zotovych is a scientific officer of UNESCO International Research Center of Information Technologies and Systems. He graduated from the mechanico - mathematical department of Kyiv State University named by T. G. Shevchenko. He worked in different institutions of Academy of Sciences of Ukraine such as Institute of Mechanics, Institute of Cybernetics named by V. Glushkov, UNESCO International Research Center of Information Technologies and Systems. He is an inventor, the author of the new technique of electro-acupuncture diagnostics. The field of his scientific investigations is mathematical modeling in biology, phisiology and medicine; non-ordinary applications of general theory of relativity, in particular for description of fields of living organisms; artificial intelligence. 


\section{Kravchenko Volodymyr Pavlovych is Ph.D. in Physics and Mathematics, senior scientific officer, scientific secretary of Engineering and Innovation Committee of Aerospace Association of Ukraine. He graduated from the mechanico-mathematical \\ department of Kyiv State University named by T. G. Shevchenko. He worked in such institutions of Academy of Sciences of Ukraine as Institute of Mechanics, Institute of Hydromechanics, Institute of Geotechnic Mechanics, Institute of Cybernetics named by V. Glushkov. He is the author of 150 scientific papers and 6 monographs.}

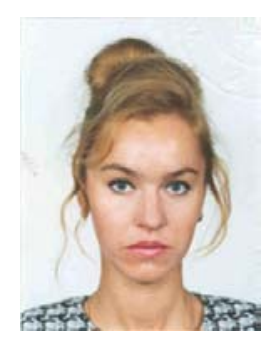

Mul Olena Vladlenivna is Ph.D. in Physics and Mathematics, associate professor of specialized computer systems department of Institute of Computer Information Technologies in Ternopil Academy of National Economy. She graduated from State university

"Lvivska Politechnika" by applied mathematics speciality. She defended Ph.D. thesis "Analysis of Self-Oscillations Processes in the Complex Continuous and Discrete Systems" at Academic Council of Space Research Institute of NASU and NSAU. She was repeatedly awarded by grants from NATO, UNESCO, CIME, IUPAP for participation in international scientific conferences and seminars.

Shut Mykola Ivanovych is a Doctor of Sciences in Physics and Mathematics, professor, head of department of general physics of National Pedagogical University (Kyiv). He is the author of more than 200 scientific papers, inventions and teaching textbooks on physics.

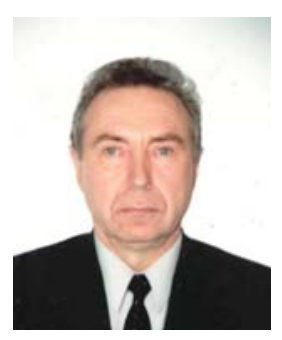

